\title{
Técnica de Pull-back para Implante de Stent em Lesões de Bifurcação com Envolvimento Único do Óstio do Ramo Lateral: Avaliação dos Resultados Iniciais e aos Seis Meses Pós-implante
}

Luciano Maurício de Abreu Filho¹, George César Ximenes Meireles ${ }^{1}$, Antonio Artur da Cruz Forte ${ }^{1}$, Marcos Kiyoshi Sumita ${ }^{1}$, José Del Carmen Solano Aliaga ${ }^{1}$, Alessandro Pina Pedroso ${ }^{1}$, Desidério Favarato ${ }^{2}$

\section{RESUMO}

Introdução: Lesões coronárias em bifurcação ainda representam um desafio ao tratamento percutâneo, com sucesso do procedimento abaixo do esperado e altas taxas de reestenose. A técnica de pull-back é uma opção no tratamento de lesões coronárias de bifurcações que apresentam acometimento isolado do óstio de ramos laterais, embora seus resultados a médio prazo ainda não sejam conhecidos. O objetivo deste estudo foi avaliar os resultados angiográficos iniciais e aos seis meses com essa técnica em nossa casuística. Método: A técnica consiste no posicionamento de duas guias, seguido do stent no ramo lateral e do balão no vaso principal encobrindo a origem do ramo. Após a insuflação do balão no vaso principal, o stent é tracionado em direção ao vaso principal até contato com o balão e, então, liberado. Resultados: Foram tratados 37 pacientes consecutivos. Os ramos diagonais foram os mais freqüentemente abordados $(70,3 \%)$. O diâmetro de referência dos ramos laterais foi de $2,51 \pm 0,37 \mathrm{~mm}$ e a extensão da lesão, de 11,1 $\pm 3,7 \mathrm{~mm}$. A pressão de liberação dos stents no ramo lateral foi de $12,8 \pm 1,9 \mathrm{~atm}$ e a pressão de insuflação do balão no vaso principal, de $7,8 \pm 0,9 \mathrm{~atm}$. O sucesso do implante ocorreu em todos os procedimentos. Foram submetidos a coronariografia tardia $22(59,5 \%)$ pacientes, tendo sido observada reestenose no ramo lateral em nove (40,9\%) pacientes e desenvolvimento de lesões > 50\% no vaso principal em sete $(31,8 \%)$. Conclusão: Lesões isoladas localizadas no óstio de ramos laterais tratadas com stent pela técnica de pull-back apresentaram elevadas taxas de sucesso do procedimento. No seguimento a médio prazo, entretanto, observou-se elevada taxa de reestenose

\section{SUMMARY}

Pull-back Technique for Stent Implantation in Coronary Bifurcation Lesions Involving Isolated Ostial Side Branch: Early and 6-month Follow-up Results

Introduction: Bifurcated coronary lesions are still a challenge to the percutaneous treatment, with a lower than expected success rate and high rates of restenosis. The pull-back technique is an option for the treatment of coronary lesions in bifurcations that compromises exclusively the ostium of lateral branches, but its middle term results are still not known. The aim of this study is to assess the initial and 6-month angiographic results of this technique in our casuistic. Method: The technique consists in the positioning of two guide-wires, followed by the stent in the lateral branch and the balloon in the main vessel, covering the origin of the branch. After insufflating the balloon in the main vessel, the stent is pulled back towards the main vessel until it touches the balloon, and then implanted. Results: Thirty-seven consecutive patients were treated. The diagonal branches were the most commonly treated $(70.3 \%)$. The reference diameter of the lateral branches was $2.51 \pm 0.37 \mathrm{~mm}$ and the extension of the lesion $11.1 \pm$ $3.7 \mathrm{~mm}$. The release pressure of the stents within the lateral branch was $12.8 \pm 1.9 \mathrm{~atm}$ and the insufflation pressure of the balloon in the main vessel was $7.8 \pm 0.9 \mathrm{~atm}$. The stent was successfully implanted in all procedures. Twenty-two $(59.5 \%)$ patients were submitted to late coronariography and restenosis was observed in the lateral branch in 9 $(40.9 \%)$ patients, and the development of lesions > 50\% in the main vessel was observed in $7(31.8 \%)$ patients.

\footnotetext{
${ }^{1}$ Hospital Stella Maris - São Paulo, SP

${ }^{2}$ Instituto do Coração do Hospital das Clínicas da Faculdade de Medicina da Universidade de São Paulo - São Paulo, SP. Correspondência: George César Ximenes Meireles. Secretaria da Pós-graduação do IAMSPE. Avenida Ibirapuera, 981 - 6o andar sala 601 - Vila Clementino - São Paulo, SP - CEP 04029-000 Tel.: (11) 5088-8452 • E-mail: george.ximenes@terra.com.br. Recebido em: 24/8/2007 • Aceito em: 24/11/2007
} 
Abreu Filho LM, et al. Técnica de Pull-back para Implante de Stent em Lesões de Bifurcação com Envolvimento Único do Óstio do Ramo Lateral: Avaliação dos Resultados Iniciais e aos Seis Meses Pós-implante. Rev Bras Cardiol Invas. 2008;16(1):44-49.

no ramo lateral e alto risco de desenvolvimento de uma nova obstrução no vaso principal.

DESCRITORES: Contenedores. Angioplastia transluminal percutânea coronária. Coronariopatia.
Conclusion: Isolated lesions found in the ostial of lateral branches treated with the "pull-back" stent implantation technique presented high rates of procedural success. However, in the middle term follow-up, a high restenosis rate in the lateral branch was observed, as well as a high risk of developing a new obstruction in the main vessel.

DESCRIPTORS: Stents. Angioplasty, transluminal, percutaneous coronary. Coronary disease.
A s lesões coronárias localizadas em bifurcações permanecem um desafio para os cardiologistas intervencionistas ${ }^{1,2}$, principalmente as lesões isoladas em óstios de ramos laterais (tipo $4 b$ de Lefèvre ${ }^{3}$ ). O tratamento dessas lesões somente com balão tem sido associado a baixas taxas de sucesso, maior risco de complicações e resultado da dilatação luminal subótimo em decorrência de recolhimento elástico agudo ${ }^{4,5}$, ocasionado pela maior concentração de fibras elásticas e musculares ao redor do óstio ${ }^{6}$. Tentativas de solução desse problema com o emprego de laser ou aterectomia rotacional foram desapontadoras $^{7,8}$. O implante de stent eliminou o recolhimento elástico, mas pode ocasionar deslocamento de placa para o vaso principal, necessitando de implante de stent adicional ${ }^{9-11}$.

A técnica de pull-back tem sido descrita como alternativa para o tratamento dessas lesões ${ }^{12}$, pois a presença do balão insuflado no vaso principal durante o implante de stent no ramo lateral pode evitar o deslocamento de placa. São desconhecidos os resultados angiográficos a médio prazo dessa técnica com o emprego de stents convencionais em lesões tipo $4 \mathrm{~b}$ de Lefèvre.

Testamos a hipótese de que o emprego da técnica de pull-back para o implante de stent em lesões isoladas em óstios de ramos laterais evita o deslocamento de placa para o vaso principal e, conseqüentemente, o uso de stent adicional, melhorando os resultados angiográficos iniciais e aos seis meses pós-implante nessas lesões.

\section{MÉTODO}

Trata-se de um registro prospectivo e unicêntrico do implante de stent pela técnica de pull-back, realizado entre novembro de 2005 e novembro de 2006, com inclusão consecutiva de 37 pacientes apresentando lesões isoladas localizadas no óstio de ramos laterais (tipo $4 \mathrm{~b}$ de Lefèvre) e portadores de doença arterial coronária com indicação de implante de stent.

O protocolo de estudo, aprovado pelo Comitê de Ética em Pesquisa da instituição, foi conduzido de acordo com os princípios da Declaração de Helsinque. Todos os pacientes assinaram o termo de consentimento livre e esclarecido.

Os procedimentos foram indicados por cardiologistas clínicos, com base na apresentação clínica e/ou no teste provocativo de isquemia miocárdica. Assim, foram incluídos pacientes com doença arterial coronária portadores de angina estável ou síndrome coronária aguda com indicação de revascularização percutânea com implante de stent coronário. Os critérios de inclusão angiográfica foram a presença de lesões de novo em bifurcações tipo $4 \mathrm{~b}$ de Lefèvre, com comprometimento único do óstio de ramo lateral, com porcentual de estenose em diâmetro $>50 \%$, ângulo do ramo lateral em relação ao vaso principal > 75 graus e diâmetro de referência $\geq 2 \mathrm{~mm}$, passíveis de tratamento pela intervenção coronária percutânea. $\mathrm{O}$ vaso principal deveria apresentar diâmetro de referência $>2,5 \mathrm{~mm}$ e ausência de qualquer obstrução em toda sua extensão. Foram excluídos pacientes com contra-indicação ao uso de aspirina e clopidogrel, pacientes submetidos a angioplastia primária, pacientes portadores de doença sistêmica crônica capaz de influenciar a sobrevida (insuficiência hepática ou renal crônica, neoplasia maligna, doença cerebrovascular e doença do tecido conjuntivo) e lesão no vaso principal.

A técnica de pull-back já foi descrita anteriormente $^{12}$ e consiste no posicionamento de duas guias metálicas, uma no ramo lateral e outra no vaso principal, confirmado por fluoroscopia. A pré-dilatação da lesão do ramo lateral ficou a critério do operador, com a utilização de balão semicomplascente $0,5 \mathrm{~mm}$ menor que o diâmetro de referência. Após a pré-dilatação, o stent foi avançado no ramo lateral, distal ao óstio. Um balão foi posicionado no vaso principal (razão nominal balão/artéria 1:1), cobrindo a origem do ramo lateral. Após a insuflação do balão no vaso principal com aproximadamente $8 \mathrm{~atm}$, o stent foi tracionado em direção ao vaso principal até que o contato com o balão ocasionasse leve depressão no balão e, então, o stent foi liberado com pressão maior ou igual a $12 \mathrm{~atm}$. A deflação dos balões podia ser realizada simultânea ou seqüencialmente, de acordo com a preferência do operador (Figura 1).

O sucesso angiográfico foi definido como a obtenção do porcentual de estenose em diâmetro infe- 
Abreu Filho LM, et al. Técnica de Pull-back para Implante de Stent em Lesões de Bifurcação com Envolvimento Único do Óstio do Ramo Lateral: Avaliação dos Resultados Iniciais e aos Seis Meses Pós-implante. Rev Bras Cardiol Invas. 2008;16(1):44-49.
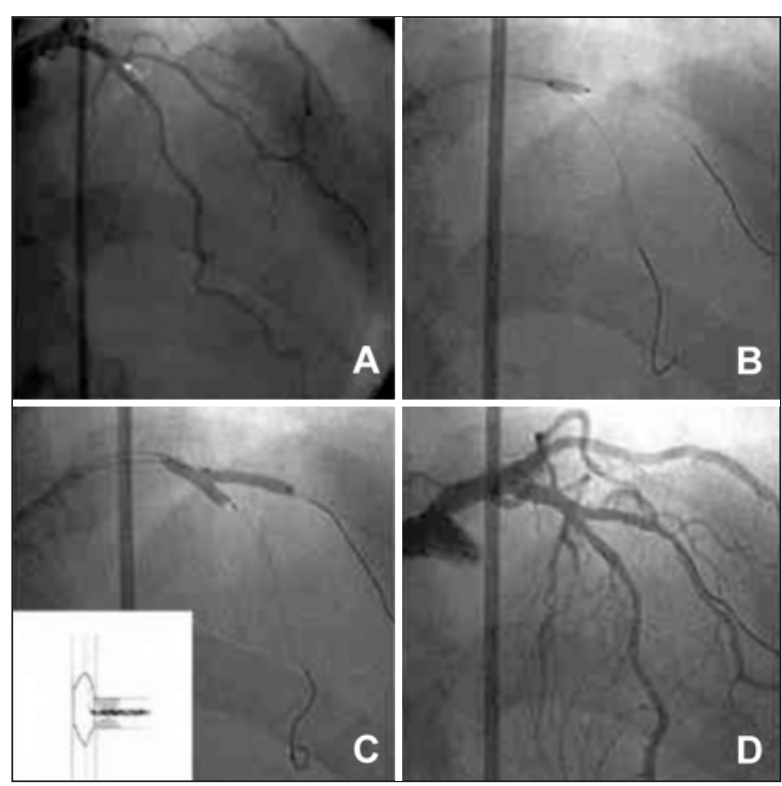

Figura 1 - Técnica de pull-back em artéria descendente anterior/ramo diagonal. A: Estenose isolada no óstio do ramo diagonal. B: Posicionamento das duas guias e pré-dilatação da lesão. C: Liberação do stent no óstio do ramo diagonal pela técnica de pull-back com balão insuflado na artéria descendente anterior. D: Resultado final.

rior a 20\% com fluxo TIMI 3 no ramo lateral, e ausência de obstruções no vaso principal. O sucesso clínico foi definido pela obtenção do sucesso angiográfico na ausência de eventos cardíacos maiores (morte cardíaca, infarto agudo do miocárdio ou cirurgia de emergência). O diagnóstico de infarto pós-implante era feito: 1. quando houvesse ondas $Q$ anormais, em duas derivações contíguas, que explorassem a mesma parede, não presentes no eletrocardiograma inicial do paciente; e 2. quando houvesse elevação enzímica maior que três vezes o limite superior normal da creatina fosfoquinase e de sua fração $M B$.

A análise coronária quantitativa foi realizada nos dois ramos, on line, utilizando a técnica de detecção semi-automática de bordas por meio do software do aparelho Philips Integris 3000 (Philips - Holanda), com o cateter-guia sem contraste como referência. Por meio desse sistema computadorizado de análise, determinou-se, em cada uma das três angiografias (pré-procedimento, imediatamente após e aos seis meses pósprocedimento), diâmetro de referência interpolado, diâmetro luminal mínimo e porcentual de estenose em diâmetro do vaso principal e do ramo lateral. $\mathrm{Na}$ angiografia pré-procedimento, foi também medida a extensão da lesão-alvo pelo mesmo sistema de análise e o ângulo do ramo lateral em relação ao vaso principal por avaliação visual (> 75 graus).

A creatina fosfoquinase fração $M B$ foi dosada entre seis e oito horas e 12 a 24 horas pós-procedimento. Todos os pacientes receberam aspirina $200 \mathrm{mg}$ por via oral, e clopidogrel em dose de ataque de $300 \mathrm{mg}$ no dia anterior ao implante de stent seguido de $75 \mathrm{mg}$ por dia durante 30 dias, exceto para o stent farmacológico (seis meses). O uso de inibidor de glicoproteína Ilb/IIla ficou a critério do operador.

Os pacientes foram reavaliados aos seis meses pós-implante de stent e eventos tais como morte, infarto agudo do miocárdio e novas revascularizações do vaso-alvo foram anotados. Na ocasião dessa avaliação, foi realizada coronariografia de controle.

A reestenose angiográfica foi definida como presença de porcentual de estenose em diâmetro > 50\% dentro do stent ou até $5 \mathrm{~mm}$ proximal e distal à área do stent. A reestenose foi categorizada como difusa quando sua extensão era maior que $10 \mathrm{~mm}$.

As variáveis quantitativas foram apresentadas como médias e desvios padrão e analisadas pelo teste $t$ de Student; as variáveis qualitativas foram apresentadas como freqüências absolutas e porcentuais e comparadas pelo teste do qui-quadrado ou teste exato de Fischer, quando apropriado.

\section{RESULTADOS}

A média das idades foi de 59,9 anos, com predomínio de pacientes do sexo feminino (54\%). A maioria $(73 \%)$ dos pacientes apresentava angina estável (Tabela 1).

A fração de ejeção foi de $60,8 \pm 13,4 \%$. Houve predominância de acometimento das bifurcações descendente anterior/diagonal (70,3\%) e circunflexa/obtuso marginal (16,2\%) (Tabela 2). A maioria (56,8\%) dos pacientes apresentava comprometimento de apenas uma artéria (uniarterial).

O sucesso do implante ocorreu em todos os procedimentos, sem complicações no vaso principal. Foi utilizado cateter-guia $7 \mathrm{~F}$ em todos os casos e a via

TABELA 1

Características clínicas basais

\begin{tabular}{lc}
\hline Variáveis & $\mathbf{n}=\mathbf{3 7}$ \\
\hline Média de idade & $59,9 \pm 10,3$ anos \\
Sexo feminino & $20(54 \%)$ \\
Diabetes melito & $7(18,9 \%)$ \\
Tabagismo & $18(48,6 \%)$ \\
Dislipidemia & $12(32,4 \%)$ \\
Hipertensão arterial sistêmica & $25(67,6 \%)$ \\
Infarto do miocárdio prévio & $5(13,5 \%)$ \\
Quadro clínico & $27(73 \%)$ \\
$\quad$ Angina estável & $10(27 \%)$ \\
\multicolumn{2}{c}{ Síndrome coronária aguda } \\
\hline $\mathrm{n}=$ número de pacientes.
\end{tabular}


Abreu Filho LM, et al. Técnica de Pull-back para Implante de Stent em Lesões de Bifurcação com Envolvimento Único do Óstio do Ramo Lateral: Avaliação dos Resultados Iniciais e aos Seis Meses Pós-implante. Rev Bras Cardiol Invas. 2008;16(1):44-49.

utilizada foi a femoral. A pressão de liberação dos stents no ramo lateral foi de $12,8 \pm 1,9$ atm e a pressão de insuflação do balão no vaso principal, de 7,8 $\pm 0,9 \mathrm{~atm}$. O diâmetro do stent foi de 2,49 $\pm 0,4 \mathrm{~mm}$ e a extensão, de 11,5 $\pm 3,6 \mathrm{~mm}$ (Tabela 3). O diâmetro do balão no vaso principal foi de 3,16 $\pm 0,41 \mathrm{~mm}$ e a extensão, de 13,6 2 2,3 mm. Todos os stents utilizados foram convencionais, à exceção de um stent farmacológico (CoStar, Conor Medsystems).

Pela angiografia coronária quantitativa, o diâmetro dos ramos laterais foi de 2,51 $\pm 0,37 \mathrm{~mm}$ e a extensão da lesão, de 11,1 $\pm 3,7 \mathrm{~mm}$. O diâmetro do vaso principal foi de $3,17 \pm 0,42 \mathrm{~mm}$ e as artérias estavam isentas de lesões obstrutivas. Foram submetidos a coronariografia tardia 22 (59,5\%) dos 37 pacientes, tendo sido observada reestenose no ramo lateral em nove $(40,9 \%)$, com predomínio do padrão difuso

TABELA 2

Características angiográficas

\begin{tabular}{|c|c|}
\hline Variáveis & $\mathbf{n}=37$ \\
\hline \multicolumn{2}{|l|}{ Função ventricular } \\
\hline Fração de ejeção & $60,8 \pm 13,4 \%$ \\
\hline \multicolumn{2}{|l|}{ Lesões } \\
\hline Característica das lesões & Tipo B2 \\
\hline \multicolumn{2}{|l|}{ Ramos das bifurcações } \\
\hline DA/diagonal & $26(70,3 \%)$ \\
\hline CX/obtuso marginal & $6(16,2 \%)$ \\
\hline CD distal VP/DP & $5(13,5 \%)$ \\
\hline \multicolumn{2}{|c|}{$\begin{array}{l}\mathrm{n}=\text { número de pacientes; } \mathrm{DA}=\text { artéria descendente anterior, } \\
\mathrm{CX}=\text { artéria circunflexa; } \mathrm{CD}=\text { artéria coronária direita; } \mathrm{VP}= \\
\text { artéria ventricular posterior; } \mathrm{DP}=\text { artéria descendente pos- } \\
\text { terior. }\end{array}$} \\
\hline
\end{tabular}

TABELA 3

Resultados do procedimento

\begin{tabular}{lc}
\hline Medidas nominais dos stents & $\mathbf{n}=\mathbf{3 7}$ \\
\hline Diâmetro do stent & $2,49 \pm 0,4 \mathrm{~mm}$ \\
Extensão do stent & $11,5 \pm 3,6 \mathrm{~mm}$ \\
Pressão de insuflação do balão no & \\
VP e de liberação dos stents no RL & \\
VP & $7,8 \pm 0,9 \mathrm{~atm}$ \\
RL & $12,8 \pm 1,9 \mathrm{~atm}$ \\
Procedimento no ramo lateral & $7(18,9 \%)$ \\
Pré-dilatação & $1(2,7 \%)$ \\
Pós-dilatação & $100 \%$ \\
Sucesso & \\
\hline $\mathrm{n}=$ número de pacientes; VP = vaso principal; RL $=$ ramo \\
lateral.
\end{tabular}

$(88,9 \%)$ (Tabela 4). Desenvolvimento de novas lesões $>50 \%$ no vaso principal foi encontrado em sete $(31,8 \%)$ pacientes. A perda tardia de foi 1,03 $\pm 0,83 \mathrm{~mm}$ e de $0,56 \pm 0,99 \mathrm{~mm}$ no ramo lateral e no vaso principal, respectivamente.

Dentre os sete pacientes com lesão $>50 \%$ no vaso principal, um apresentava lesão isolada na artéria descendente anterior, no local da insuflação do balão, e foi submetido a cirurgia de revascularização miocárdica. Os demais pacientes apresentavam lesões envolvendo o vaso principal e o ramo lateral, dos quais três foram submetidos a cirurgia de revascularização miocárdica e três, a implante de stent no vaso principal e angioplastia com balão no ramo lateral.

O seguimento clínico foi obtido em todos os pacientes, tendo sido observado que $26(70,3 \%)$ pacientes evoluíram assintomáticos e 10 (27\%) apresentaram angina aos grandes esforços. Ocorreu um óbito de causa não-cardíaca e não foram observados casos de trombose de stent.

\section{DISCUSSÃO}

Trata-se da primeira publicação na literatura médica com resultados angiográficos imediatos e tardios após o emprego da técnica de pull-back para o tratamento de lesões localizadas no óstio de ramos laterais (tipo $4 \mathrm{~b}$ de Lefèvre), que tem como objetivos a cobertura completa dessas lesões pelo stent e evitar o deslocamento de placa e o conseqüente implante de stent não planejado no vaso principal.

Os resultados imediatos dos implantes dos stents no presente estudo confirmaram que a técnica de pullback, quando avaliada pela angiografia digital, não demonstra deslocamento de placa e que o barotrauma no vaso principal, no momento do implante, não acarretou complicações, pois o sucesso ocorreu em todos os procedimentos e não foi necessário implante de stents adicionais. O mesmo não ocorreu em relação aos resultados a médio prazo, que demonstraram que o balão insuflado com pressão nominal de $8 \mathrm{~atm}$ no vaso principal pode ocasionar lesão obstrutiva em artéria sem lesão prévia, observando-se a presença de obstrução > 50\% no vaso principal em $31,8 \%$ dos pacientes submetidos a coronariografia aos seis meses pós-implante.

O único estudo publicado sobre a efetividade da técnica de pull-back no tratamento dessas lesões, em comparação ao uso da técnica convencional com prédilatação com balão ou cutting balloon, demonstrou que durante o procedimento houve necessidade de stents adicionais em $2 \%$ vs. $18 \%$ dos pacientes e que a taxa de revascularização do vaso-alvo foi de $5 \%$ vs. $20 \%$, respectivamente. Esse estudo avaliou apenas a reestenose clínica, e as angiografias foram realizadas com base somente na manifestação clínica de isquemia miocárdica, o que deve ter contribuído para os núme- 
Abreu Filho LM, et al. Técnica de Pull-back para Implante de Stent em Lesões de Bifurcação com Envolvimento Único do Óstio do Ramo Lateral: Avaliação dos Resultados Iniciais e aos Seis Meses Pós-implante. Rev Bras Cardiol Invas. 2008;16(1):44-49.

TABELA 4

Medidas angiográficas pré, imediatamente após e seis meses pós-procedimento de implante de stent em ramo lateral e vaso principal

\begin{tabular}{lcc} 
& Ramo lateral & Vaso principal \\
\hline Pré-implante & $\mathrm{n}=37$ & $\mathrm{n}=37$ \\
Diâmetro de referência & $2,51 \pm 0,37 \mathrm{~mm}$ & $3,17 \pm 0,42 \mathrm{~mm}$ \\
Diâmetro luminal mínimo & $0,44 \pm 0,12 \mathrm{~mm}$ & $3,16 \pm 0,42 \mathrm{~mm}$ \\
Porcentual de estenose & $84,5 \pm 7,2 \%$ & - \\
Extensão & $11,1 \pm 3,7 \mathrm{~mm}$ & - \\
Imediatamente pós-implante & $\mathrm{n}=37$ & $\mathrm{n}=37$ \\
Diâmetro de referência & $2,5 \pm 0,37 \mathrm{~mm}$ & $3,17 \pm 0,42 \mathrm{~mm}$ \\
Diâmetro luminal mínimo & $2,45 \pm 0,54 \mathrm{~mm}$ & $3,17 \pm 0,42 \mathrm{~mm}$ \\
Porcentual de estenose & $3,1 \pm 1,1 \%$ & - \\
Seis meses pós-implante & $\mathrm{n}=22$ & $\mathrm{n}=22$ \\
Diâmetro de referência & $2,51 \pm 0,46 \mathrm{~mm}$ & $3,2 \pm 0,42 \mathrm{~mm}$ \\
Diâmetro luminal mínimo & $1,48 \pm 0,97 \mathrm{~mm}$ & $2,23 \pm 1,14 \mathrm{~mm}$ \\
Porcentual de estenose & $44,4 \pm 35,8 \%$ & $27,3 \pm 34,9 \%$ \\
Taxa de reestenose & $9 / 22(40,9 \%)$ & - \\
\hline
\end{tabular}

$\mathrm{n}=$ número de pacientes.

ros bastante favoráveis dessa técnica. Vale ressaltar que, em nosso estudo, dentre os sete pacientes que apresentaram progressão de lesões > 50\% no vaso principal, a maioria (70\%) encontrava-se assintomática. Adicionalmente, as pressões de insuflação do balão no vaso principal também diferiram: pressões mais baixas (4 a 6 atm) foram utilizadas no primeiro caso, enquanto em nosso estudo foram empregadas pressões de $8 \mathrm{~atm}^{12}$. Empregamos a relação balão/artéria 1:1 e pressões nominais do balão mais altas, com a intenção de tracionar o sistema balão/stent posicionado no ramo lateral em direção ao vaso principal, evitando o deslocamento de placa para o vaso principal e permitindo a localização precisa da extremidade proximal do stent no óstio do ramo lateral. Essa estratégia, no entanto, pode ter contribuído para o impacto negativo em nossos resultados.

Uma grande limitação da técnica de pull-back é sua utilização somente nos casos em que o ângulo do ramo lateral é maior que 75 graus. Nos casos com ângulos menores, há maior probabilidade de a porção superior do óstio do ramo não ficar adequadamente coberta pelo stent ou os stents invadirem o vaso principal $^{12}$.

Muitas vezes o tratamento de lesões no óstio de ramos laterais (tipo $4 \mathrm{~b}$ de Lefèvre) por meio do implante de stent convencional, único dispositivo disponível para pacientes provenientes do Sistema Único de Saúde (SUS), é evitado em decorrência das elevadas taxas de reestenose. No presente estudo, a manifestação clínica predominante foi a angina estável, mas em $10(27 \%)$ dos 37 pacientes incluídos no estudo a apresentação foi como síndrome coronária aguda (seis casos de angina instável e quatro casos de infarto agudo do miocárdio, três deles com supradesnivelamento de ST), evidenciando a importância do desenvolvimento de procedimentos alternativos para o tratamento dessas lesões.

Essa técnica aplicada aos stents farmacológicos, que se mostraram eficazes na redução da reestenose quando implantados em lesões localizadas em bifurcações ${ }^{13,14}$, tem o potencial de evitar a cobertura inadequada do óstio de ramos laterais, reduzir o uso de stents adicionais pelo deslocamento de placa para o vaso principal e reduzir a taxa de reestenose. No presente estudo, foi implantado apenas um stent farmacológico, pois a quase totalidade dos pacientes era proveniente do SUS, que não autoriza o emprego desses stents.

As limitações do presente estudo foram: não utilização do ultra-som intracoronário, que poderia contribuir para a avaliação da expansão e da cobertura completa do stent no óstio do ramo lateral; ausência de grupo controle com implante pela técnica tradicional; e seguimento angiográfico aos seis meses pósimplante limitado a $60 \%$ dos pacientes, inferior ao preconizado $(>80 \%)$ para avaliar a presença de reestenose como desfecho principal.

\section{CONCLUSÃO}

Nossos resultados demonstraram que o implante de stent convencional em lesões isoladas localizadas no óstio de ramos laterais pela técnica de pull-back apresenta elevadas taxas de sucesso do procedimento. 
Abreu Filho LM, et al. Técnica de Pull-back para Implante de Stent em Lesões de Bifurcação com Envolvimento Único do Óstio do Ramo Lateral: Avaliação dos Resultados Iniciais e aos Seis Meses Pós-implante. Rev Bras Cardiol Invas. 2008;16(1):44-49.

Entretanto, no seguimento a médio prazo, foi observada elevada taxa de reestenose no ramo lateral e alto risco de desenvolvimento de obstrução no vaso principal previamente livre de lesões.

\section{REFERÊNCIAS BIBLIOGRÁFICAS}

1. Pinkerton CA, Slack JD, Van Tassel JW, Orr CM. Angioplasty for dilatation of complex coronary artery bifurcation stenoses. Am J Cardiol. 1985;55(13 pt 1):1626-8.

2. Mathias DW, Mooney JF, Lange HW, Goldenberg IF, Gobel $\mathrm{FL}$, Mooney MR. Frequency of success and complications of coronary angioplasty of a stenosis at the ostium of a branch vessel. Am J Cardiol. 1991;67(6):491-5.

3. Lefevre T, Louvard Y, Morice MC, Dumas P, Loubeyre C, Benslimane A, et al. Stenting of bifurcation lesions: classification, treatments, and results. Catheter Cardiovasc Interv. 2000;49(3):274-83.

4. Finci L, Meier B, Divernois J. Percutaneous transluminal coronary angioplasty of a bifurcation narrowing using the kissing wire monorail balloon technique. Am J Cardiol. 1987;60(4):375-6.

5. Ciampricotti R, el Gamal M, Van Gelder B, Bonnier J, Taverne R. Coronary angioplasty of bifurcational lesions without protection of large side branches. Cathet Cardiovasc Diagn. 1992;27(3):191-6.

6. Kawakami $H$, Matsuoka $H$, Koyama $Y$, Saeki $H$, Inoue $K$, Nishimura K, et al. Isolated left coronary ostial stenosis as a result of fibromuscular dysplasia in a young man. Jpn Circ J. 2000;64(12):988-9.

7. Koller PT, Freed M, Grines CL, O'Neill WW. Success, complications, and restenosis following rotational and transluminal extraction atherectomy of ostial stenoses. Cathet Cardiovasc Diagn. 1994;31(4):255-60.

8. Eigler NL, Weinstock B, Douglas JS Jr, Goldenberg T, Hartzler G, Holmes D, et al. Excimer laser coronary angioplasty of aorto-ostial stenoses. Results of the excimer laser coronary angioplasty (ELCA) registry in the first 200 patients. Circulation. 1993;88(5 pt 1):2049-57.

9. Pan M, Suarez de Lezo J, Medina A, Romero M, Hernández E, Segura J, et al. Simple and complex stent strategies for bifurcated coronary arterial stenosis involving the side branch origin. Am J Cardiol. 1999;83(9):1320-5.

10. Castriz JL, Canales ML, Reynolds DW. Kissing balloon technique in complex PTCA: single guiding catheter and dual wire rapid exchange system. Cathet Cardiovasc Diagn. 1993;28(4):358-60.

11. Dardas PS, Tsikaderis DD, Mezilis NE, Styliadis G. A technique for type 4 a coronary bifurcation lesions: initial results and 6-month clinical evaluation. J Invasive Cardiol. 2003;15(4):180-3.

12. Kini AS, Moreno PR, Steinheimer AM, Prattipati M, Suleman J, Kim MC, et al. Effectiveness of the stent pull-back technique for nonaorto ostial coronary narrowings. Am J Cardiol. 2005;96(8):1123-8.

13. Colombo A, Moses JW, Morice MC, Ludwig J, Holmes DR Jr, Spanos V, et al. Randomized study to evaluate sirolimus-eluting stents implanted at coronary bifurcation lesions. Circulation. 2004;109(10):1244-9.

14. Pan M, de Lezo JS, Medina A, Romero M, Segura J, Pavlovic $D$, et al. Rapamycin-eluting stents for the treatment of bifurcated coronary lesions: a randomized comparison of a simple versus complex strategy. Am Heart J. 2004;148(5):857-64. 